\title{
Linking osteoporosis with Alzheimer disease
}

Alzheimer disease is frequently associated with fractures and reductions in bone mineral density, even at early stages of disease when patients are still active. However, the mechanisms underlying this link are unclear. Using mouse models, Wen-Cheng Xiong and colleagues have now provided some insight into this connection, by identifying a cell-autonomous defect in osteoblast differentiation, as published in the Journal of Bone and Mineral Research.

In preliminary experiments, the investigators confirmed the expression of amyloid precursor protein (APP) in bone marrow stromal cells (BMSCs) from wild-type mice, and showed that the levels of this protein increased with age. Next, they used transgenic mice expressing an Alzheimer diseaseassociated mutant form of APP, known as APPswe, to explore the effects of mutant APP on bone. From a young age, these mice displayed defects in bone formation compared with wildtype controls. Moreover, a concomitant increase in adipocytes was observed in their bone marrow, resembling a skeletal ageing phenotype.

\section{4 ...Alzheimer disease and osteoporosis may share conserved pathogenic mechanisms... 77}

In accordance with these findings, the differentiation of APPswe-transgenic BMSCs into osteoblasts was reduced in ex vivo culture experiments, whereas adipocyte generation was enhanced, suggesting that the presence of APPswe skews BMSC differentiation towards the adipocyte lineage. But how does APPswe exert this effect?

Given the key part oxidative stress is thought to play in the pathogenesis of Alzheimer disease, the researchers examined whether an antioxidant could reduce the bone phenotypes of the transgenic mice. Indeed, treatment with $N$-acetylcysteine ameliorated the impact of APPswe on BMSC differentiation, and increased bone volume in the transgenic mice. Thus, "reactive oxygen species may be a conserved mechanism underlying APPswe-induced neurodegenerative and osteoporotic deficits," suggests Xiong.

Finally, similar bone loss and diminished osteoblast formation were observed in mice selectively expressing APPswe in mature osteoblasts, demonstrating that the skeletal effects of mutant APP are likely to be, at least in part, osteoblast autonomous.

"These results support the view that Alzheimer disease risk genes may also be a risk factor for osteoporosis, and that Alzheimer disease and osteoporosis may share conserved pathogenic mechanisms," concludes Xiong, who plans to further test such ideas in future work.

Isabel Woodman

Original article: Xia, W.-F. et al. Swedish mutant APP suppresses osteoblast differentiation and causes osteoporotic deficit, which are ameliorated by N-acetyl-Lcysteine. J. Bone Miner. Res. 28, 2122-2135 (2013) 